\title{
Inhibition of hypoxia-induced epithelial mesenchymal transition by luteolin in non-small cell lung cancer cells
}

\author{
JUNSHAN RUAN $^{1,3^{*}}$, LEI ZHANG $^{1 *}$, LINGGENG YAN $^{1}$, YUPING LIU $^{1}$, ZHIQIANG YUE $^{1}$, LI CHEN $^{1}$, \\ AI-YUN WANG ${ }^{1}$, WENXING CHEN ${ }^{1}$, SHIZHONG ZHENG ${ }^{1}$, SHAOMING WANG ${ }^{3}$ and YIN LU $^{1,2}$ \\ ${ }^{1}$ Department of Clinical Pharmacy, College of Pharmacy; ${ }^{2}$ Jiangsu Key Laboratory for Pharmacology and Safety Evaluation of \\ Chinese Materia Medica, Nanjing University of Chinese Medicine, Nanjing, Jiangsu 210029; \\ ${ }^{3}$ Fujian Provincial Hospital, Clinical College of Fujian Medical University, Fuzhou, Fujian 350001, P.R China
}

Received January 21, 2012; Accepted April 16, 2012

DOI: $10.3892 / \mathrm{mmr} .2012 .884$

\begin{abstract}
Hypoxia-induced epithelial mesenchymal transition (EMT) is an essential step in cancer metastasis. Luteolin, a flavonoid that is widely distributed in plants, is a novel anticancer agent. However, the mechanism underlying its anticancer effects remains undefined.In this study, for the first time, we demonstrate that luteolin inhibits hypoxia-induced EMT in human non-small cell lung cancer cells in culture, which is demonstrated by the fact that hypoxia-induced EMT reduced the expression of E-cadherin and other epithelial markers and increased the expression of $\mathrm{N}$-cadherin, vimentin and other mesenchymal markers; these effects were markedly attenuated by luteolin. In addition, luteolin also inhibited hypoxia-induced proliferation, motility and adhesion in the cells. Furthermore, we reveal that luteolin inhibits the expression of integrin $\beta 1$ and focal adhesion kinase (FAK).Since integrin $\beta 1$ and FAK signaling are closely related to EMT formation, these results suggest that luteolin inhibits hypoxia-induced EMT, at least in part, by inhibiting the expression of integrin $\beta 1$ and FAK.
\end{abstract}

\section{Introduction}

Lung cancer is one of the most frequently occurring malignancies (1). The diagnosis of lung cancer falls into two broad categories consisting of small cell lung cancer and non-small

Correspondence to: Dr Yin Lu, Jiangsu Key Laboratory for Pharmcology and Safety Evaluation of Chinese Materia Medica, Nanjing University of Chinese Medicine, 282 Hanzhong Road, Nanjing, Jiangsu 210029, P.R. China

E-mail: profyinlu@163.com

${ }^{*}$ Contributed equally

Abbreviations: EMT, epithelial mesenchymal transition; FAK, focal adhesion kinase; NSCLC, non-small cell lung cancer

Key words: luteolin, epithelial mesenchymal transition, lung cancer, hypoxia, integrin $\beta 1$, focal adhesion kinase cell lung cancer (NSCLC), with approximately $85 \%$ of the cases involving the latter. NSCLC is sufficiently aggressive that it leads to a poor prognosis (2). Currently, NSCLC is usually treated by surgery, radiotherapy and chemotherapy, depending on the cancer stage. Chemotherapy regimens for the treatment of advanced NSCLC often include cisplatin or carboplatin combined with paclitaxel, gemcitabine, etoposide, docetaxel or vinorelbine (3). However, no patient with an advanced disease stage has been effectively cured. Moreover, chemoresistance frequently occurs in NSCLC. Therefore, it is necessary to identify new therapeutic agents that are more efficient than, or will synergize with, existing anticancer drugs.

Global gene expression analysis has revealed higher mRNA expression of epithelial-mesenchymal transition (EMT) markers in NSCLC specimens, suggesting the involvement of EMT in NSCLC development (4). Hypoxia is a clinically important component of the tumor microenvironment in NSCLC due to the adverse effects of hypoxia on tumor progression, metastasis, response to chemo/radiotherapy and overall patient survival (5-7). In NSCLC, high hypoxia levels correlate with EMT (8). Therefore, targeting hypoxia-induced EMT is an alternative approach for the prevention and treatment of NSCLC.

Luteolin (3',4',5,7-tetrahydroxyflavone) is a common dietary flavonoid in plants and foods (9). Luteolin-rich plants have been utilized in traditional Chinese medicine for the treatment of hypertension, inflammatory diseases and cancer. Preclinical research has demonstrated that luteolin possesses various pharmacological activities, including antioxidant, anti-inflammatory, antimicrobial and anticancer activities $(10,11)$. Notably, luteolin can cross the blood-brain barrier, allowing its use for the treatment of central nervous system diseases, including brain tumors. As an anticancer agent, luteolin inhibits cancer cell proliferation by suppressing protein kinases and inducing apoptosis by inhibiting cell survival pathways (12). The activity of this compound regulates the redox state, causing DNA damage (13). Luteolin also inhibits metastasis and angiogenesis $(14,15)$.

Luteolin has been reported to inhibit human colon (HT-29 and HCT116), liver (SK-HEP-1, PLC/PRF/5 and Hep3), cervical (HeLa), as well as oral (SCC-4) tumor cell growth (16). However, research exploring the mechanism of action of luteolin 
in human lung cancer cells has not been reported. In addition, whether and how luteolin inhibits hypoxia-induced EMT has not been clarified. In this study, we demonstrate that luteolin inhibits hypoxia-induced proliferation, migration and adhesion in NSCLC (A549 and NCI-H1975) cells. In addition, luteolin inhibits hypoxia-induced EMT, at least in part, by inhibiting the expression of integrin $\beta 1$ and focal adhesion kinase (FAK).

\section{Materials and methods}

Reagents. Luteolin, which was purchased from Xi'an Helin Biological Engineering Co., Ltd. (Xi'an, Shaanxi, China), was dissolved in dimethylsulfoxide (DMSO) and then added to the medium at the indicated concentrations to limit the DMSO concentration to below $0.1 \%$. The antibodies against $\mathrm{N}$-cadherin, E-cadherin, integrin $\beta 1$, vimentin and GAPDH were purchased from Bioworld Technology (St. Louis Park, MN, USA), and the antibodies to FAK were purchased from Signalway Antibody (Pearland, TX, USA). The EMT sample kit was purchased from Cell Signaling Technology (Danvers, MA, USA) and the FAK inhibitor was purchased from Santa Cruz Biotechnology, Inc. (Santa Cruz, CA, USA).

Cell culture. Human A549 and NCI-H1975 (H1975) NSCLC cells (American Type Culture Collection, Manassas, VA, USA) were grown in RPMI-1640 medium (Gibco, Grand Island, NY, USA) supplemented with $10 \%$ fetal bovine serum (FBS; Gibco), 100 units $/ \mathrm{ml}$ penicillin and $100 \mu \mathrm{g} / \mathrm{ml}$ streptomycin. The cells were maintained in a humidified incubator $\left(37^{\circ} \mathrm{C}\right.$, $5 \% \mathrm{CO}_{2}$ ) and detached with $0.25 \%$ trypsin-0.02\% EDTA (Gibco). Chronic hypoxic-conditioned cells were derived by being exposed to hypoxia $\left(1 \% \mathrm{O}_{2}\right)$ for 1 week as previously described (17). All other cell culture reagents were obtained from Gibco.

3-(4,5-dimethylthiazol-2-yl)-5-(3-carboxymethoxyphenyl)-2(4-sulfophenyl)-2H-tetrazolium (MTS) assay. The effect of luteolin on nomal and hypoxic A549/H1975 cell proliferation and viability was evaluated using an MTS assay with the CellTiter $96^{\circledR} \mathrm{AQ}_{\text {ueous }}$ One Solution Cell Proliferation Assay kit (Promega, Madison, WI, USA). Briefly, exponentially growing cells were trypsinized and seeded in 96 -well plates $\left(5 \times 10^{3} /\right.$ well) in complete medium. After incubation for $24 \mathrm{~h}$, the cells were cultured under chronic hypoxic conditions and further incubated in the presence or absence of the indicated luteolin concentrations for $24 \mathrm{~h}$. Subsequently, $20 \mathrm{ml}$ of the solution reagent were added to each well. Following incubation of the plate for $3 \mathrm{~h}$ at $37^{\circ} \mathrm{C}$, the optical density (OD) was measured at $490 \mathrm{~nm}$ using a VersaMax microplate reader (Molecular Devices, Sunnyvale, CA, USA).

Cell motility assay. The cell motility assay was performed using a modified Boyden Chamber (Corning, Corning, NY, USA) equipped with a polycarbonate filter ( $8 \mu \mathrm{m}$ pores). Normal and hypoxic A549/H1975 cells were grown to $80 \%$ confluence and then treated with different luteolin concentrations for $24 \mathrm{~h}$. These cells were then serum-starved overnight in RPMI-1640 medium, trypsinized and washed in serum-free RPMI-1640 medium before seeding in Transwell chambers. After the chambers were pre-treated with RPMI-1640 medium containing $0.01 \%$ FBS and $0.01 \%$ bovine serum albumin overnight, cells $\left(5 \times 10^{5}\right)$ in $200 \mu$ of serum-free RPMI-1640 medium were added to the upper chamber; the lower chamber contained RPMI-1640 medium, 10\% FBS and various agents as referred to in the Results section. Following incubation of the chambers for an additional $8 \mathrm{~h}$, the filter was removed, and the cells remaining on the upper membrane surface were scraped off. The cells that had migrated to the lower surface were then fixed in $70 \%$ ethanol for $10 \mathrm{~min}$ and were stained with $0.5 \%$ Coomassie Brilliant Blue for $10 \mathrm{~min}$. The migrated cells from 6 randomly selected fields on each filter were visualized using an inverted microscope (magnification, x200).

Cell adhesion assay. Normal and hypoxic A549/H1975 cells were grown in 6-well plates in normal growth medium for $24 \mathrm{~h}$ and then incubated with luteolin $(5,10,25$ and $50 \mu \mathrm{M})$ plus the vehicle $\left(0.1 \% \mathrm{DMSO}\right.$, control) for $24 \mathrm{~h}$ at $37^{\circ} \mathrm{C}$. The cells were trypsinized and seeded in 96-well plates or gelatincoated 96-well plates $\left(2.5 \times 10^{4} /\right.$ well $)$ in normal growth medium. After $1 \mathrm{~h}$, the medium was discarded, and the cells were gently washed twice with phosphate-buffered saline (PBS) to remove non-attached cells, followed by Rose Bengal staining to analyze the adhesion activity. Finally, the cells were fixed with $0.5 \%$ glutaraldehyde, stained with a solution $(100 \mu \mathrm{l} /$ well $)$ containing $0.1 \%$ (w/v) crystal violet for $30 \mathrm{~min}$, and solubilized in $200 \mu \mathrm{l}$ of $30 \%(\mathrm{v} / \mathrm{v})$ acetic acid. The plates were agitated for $5 \mathrm{~min}$, and the absorbance at $570 \mathrm{~nm}$ was measured using a VersaMax microplate reader (Molecular Devices).

Western blot analysis. The cells were washed with cold PBS buffer and lysed in $10 \mathrm{mM}$ Tris- $\mathrm{HCl}, \mathrm{pH} 7.4,50 \mathrm{mM}$ $\mathrm{NaCl}, 5 \mathrm{mM}$ EDTA, $1 \%$ Nonidet P-40 and $10 \mu \mathrm{g} / \mathrm{ml}$ phenylmethylsulfonyl fluoride. Cell extracts were then transferred to microcentrifuge tubes, mixed and left on ice for $10 \mathrm{~min}$. They were then centrifuged at $12,000 \mathrm{xg}$ for $5 \mathrm{~min}$ at $4^{\circ} \mathrm{C}$ after 1 freeze/thaw cycle. The protein concentrations in the supernatants were determined using a $\mathrm{BCA}^{\mathrm{TM}}$ protein assay kit (Pierce, Rockford, IL, USA). Protein samples (50 $\mu \mathrm{g} /$ lane) were resolved by SDS-PAGE and then transferred to a polyvinylidene difluoride membrane (Millipore, Billerica, MA, USA). The blots were incubated with primary antibodies diluted with Tris-buffered saline-Tween-20 containing $2.5 \%$ dried milk overnight. After successive washes with Trisbuffered saline-Tween-20, the membranes were incubated with the corresponding secondary antibodies conjugated to horseradish peroxidase. A SuperSignal kit (Pierce) was used to visualize the immunoreactive bands according to the manufacturer's instructions.

Statistical analysis. Each experiment was conducted in triplicate. Data are expressed as the means \pm standard deviation (SD) and analyzed by one-way analysis of variance and Fisher's protected least significant difference test for multiple comparisons (SPSS 10.0, SPSS Inc., Chicago, IL, USA). P<0.05 was considered to indicate a statistically significant difference.

\section{Results}

Induction of an EMT-like morphological change in A549 cells by hypoxia. To investigate whether A549/H1975 cells would 
A

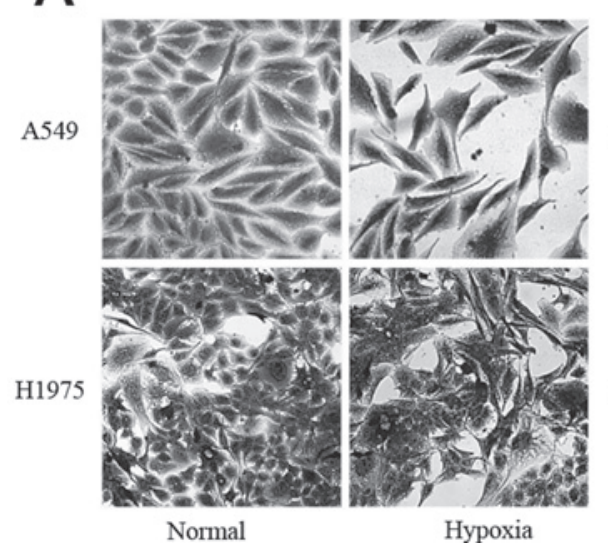

B

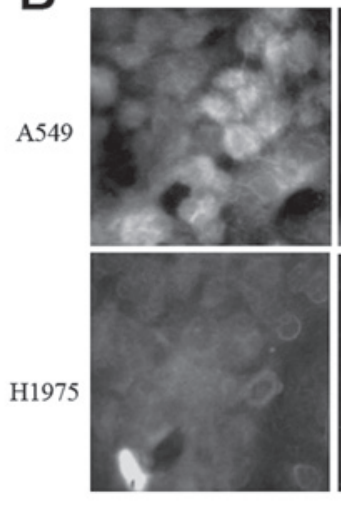

Normal

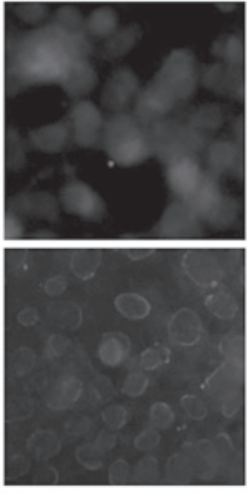

Hypoxia

Figure 1. Induction of EMT by hypoxia in A549 and NCI-H1975 cells. (A) A549 and NCI-H1975 cells were derived by being exposed to hypoxia ( $1 \% \mathrm{O}_{2}$ ) in a $37^{\circ} \mathrm{C}$ incubator for 1 week and then stained with $0.5 \%$ Coomassie Brilliant Blue for 10 min. Cells were then monitored for phenotypic changes. Cells, which typically had an epithelial phenotype with well-developed cell junctions, acquired a spindle shape and lost cell contact under hypoxic conditions. (B) Normal and hypoxic A549 and NCI-H1975 cells were exposed to $1 \% \mathrm{O}_{2}$ for 1 week, followed by fluorescence immunohistochemistry analysis to detect E-cadherin proteins. EMT, epithelial mesenchymal transition.

undergo the EMT process in response to hypoxia, the cells were cultured under chronic hypoxic conditions by being exposed to hypoxia $\left(1 \% \mathrm{O}_{2}\right)$ for 1 week and then stained with $0.5 \%$ Coomassie Brilliant Blue for 10 min to monitor the phenotypic changes. Under normal conditions, the appearance of the A549/H1975 cells were characteristically cobble-stonelike, and clusters formed when they were grown in culture due to the intercellular junctions responsible for maintaining epithelial cell adhesion and polarity (Fig. 1A).

$\mathrm{E}$-cadherin is recognized as the most common epithelial marker forEMT.EMT is characterized by the loss of E-cadherin . Therefore, an immunofluorescence assay was performed to determine the cellular protein levels of E-cadherin to verify the induction of EMT by hypoxia. Chronic hypoxic conditions reduced the expression of E-cadherin, indicating that hypoxia induced EMT in A549/H1975 cells (Fig. 1B).

Inhibition of hypoxia-induced cell adhesion, proliferation and motility by luteolin. Subsequently, we investigated the influence of luteolin on hypoxia-induced adhesion, proliferation and motility in order to clarify how luteolin functions as an anticancer agent. Consistent with the above findings, hypoxia increased cell adhesion to HUVEC-coated (Fig. 2A) and gelatincoated 96-well plates (Fig. 2B) in A549/H1975 cells. However, when the cells were incubated with luteolin $(0-50 \mu \mathrm{M})$ for $24 \mathrm{~h}$, cell adhesion was inhibited in a concentration-dependent manner (Fig. 2A and B). Additionally, we observed that luteolin not only inhibited the proliferation or reduced the cell viability of the A549/H1975 cells under hypoxic conditions (Fig. 2C) as was shown by the MTS assay, but also significantly attenuated hypoxia-induced cell motility (Fig. 2D and E).

Blocking of hypoxia-induced EMT by luteolin in A549/ H1975 cells. Taking into consideration that hypoxia-induced EMT is a key step in metastasis (8), the effect of luteolin on hypoxia-induced EMT was further examined. Normal and hypoxic A549/H1975 cells were treated with luteolin $(0-50 \mu \mathrm{M})$ for $24 \mathrm{~h}$, then an immunofluorescence assay was performed to determine the cellular protein levels of E-cadherin. Chronic hypoxic conditions reduced the expression of E-cadherin and luteolin increased the E-cadherin protein level, which indicated that luteolin blocked hypoxia-induced EMT in A549/H1975 cells (Fig. 3A).

To make the results more comprehensive, we then detected EMT-related proteins using the EMT sample kit (Cell Signaling Technology). While hypoxia markedly suppressed the expression of E-cadherin and ZO-1 (epithelial marker), and increased the expression of $\mathrm{N}$-cadherin, TCF/ZEB1 and claudin-1 (specific myofibroblast markers) and promoted $\beta$-catenin, snail and vimentin (EMT nuclear transcription factor) in hypoxic A549/H1975 cells (Fig. 3) as revealed by western blot analysis, the addition of luteolin, even at $5-10 \mu \mathrm{M}$, was able to hinder the hypoxia-induced suppression of the epithelial marker expression and attenuate that of the specific myofibroblast markers (Fig. 3).

Inhibition of expression of integrin $\beta 1$ and FAK by luteolin. As integrin and FAK signaling is critical for EMT formation $(18,19)$, we therefore hypothesized that luteolin can inhibit hypoxia-induced EMT by targeting these proteins. As expected, luteolin inhibited the expression of integrin $\beta 1$ and FAK in hypoxic A549/H1975 cells (Fig. 4A and B). The downregulation of integrin $\beta 1$ and FAK was accompanied by the upregulation of E-cadherin, suggesting that luteolin inhibits hypoxia-induced EMT, at least in part, by inhibiting the expression of integrin $\beta 1$ and FAK.

In addition, we found that FAK inhibitors did not block hypoxia-induced integrin $\beta 1$ expression, but blocked the effect of the drug on the EMT (Fig. 4C and D). The experimental results demonstrate that the effect of the drug depends on the regulation of EMT by integrin $\beta 1$.

\section{Discussion}

As a multifactorial disease, advanced cancer involves numerous genetic and epigenetic alterations influencing multiple distinct 
A

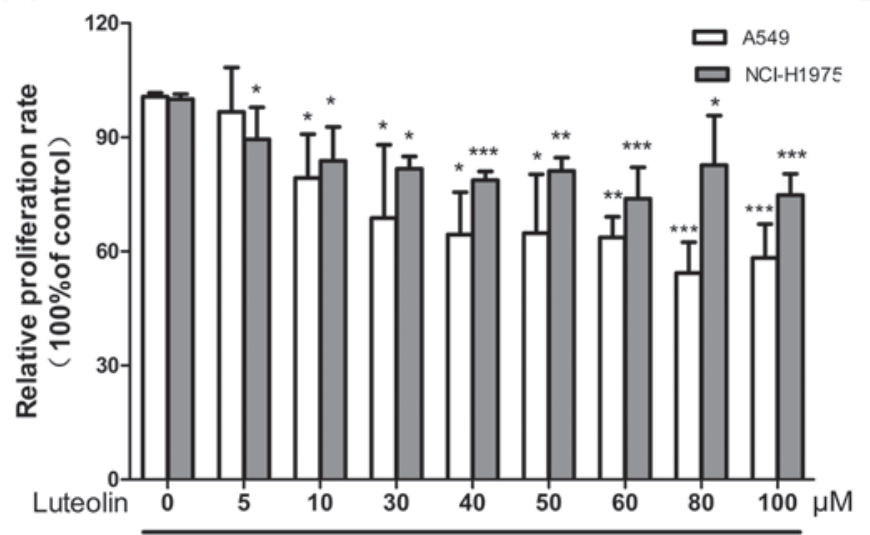

Hypoxia
B

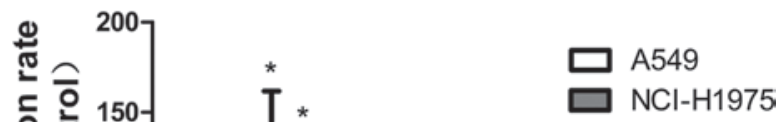

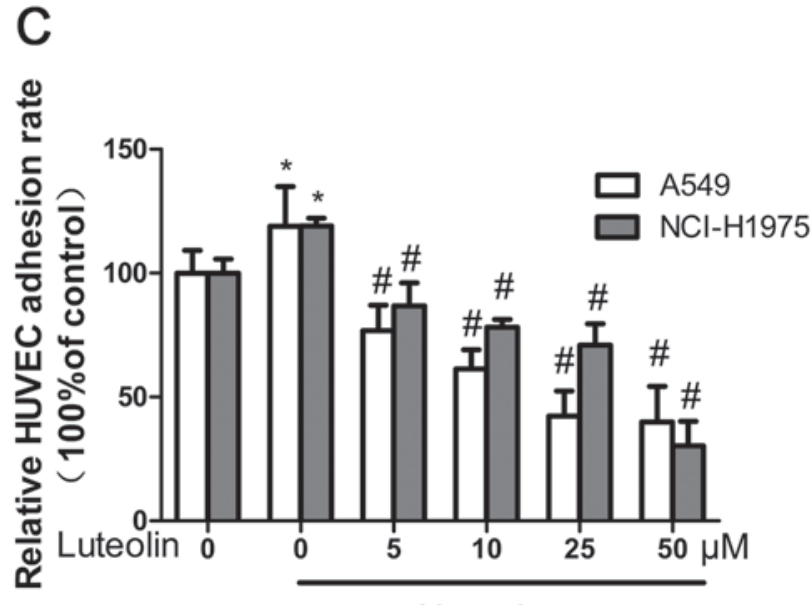

Hypoxia

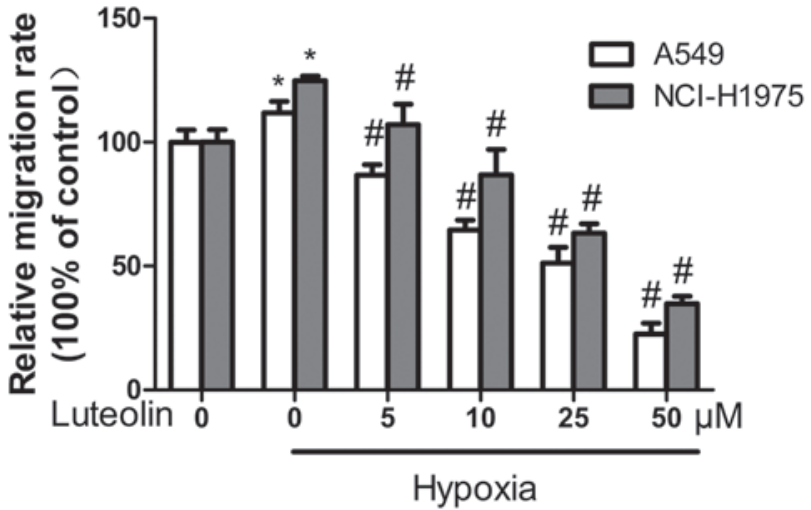

Hypoxia

E

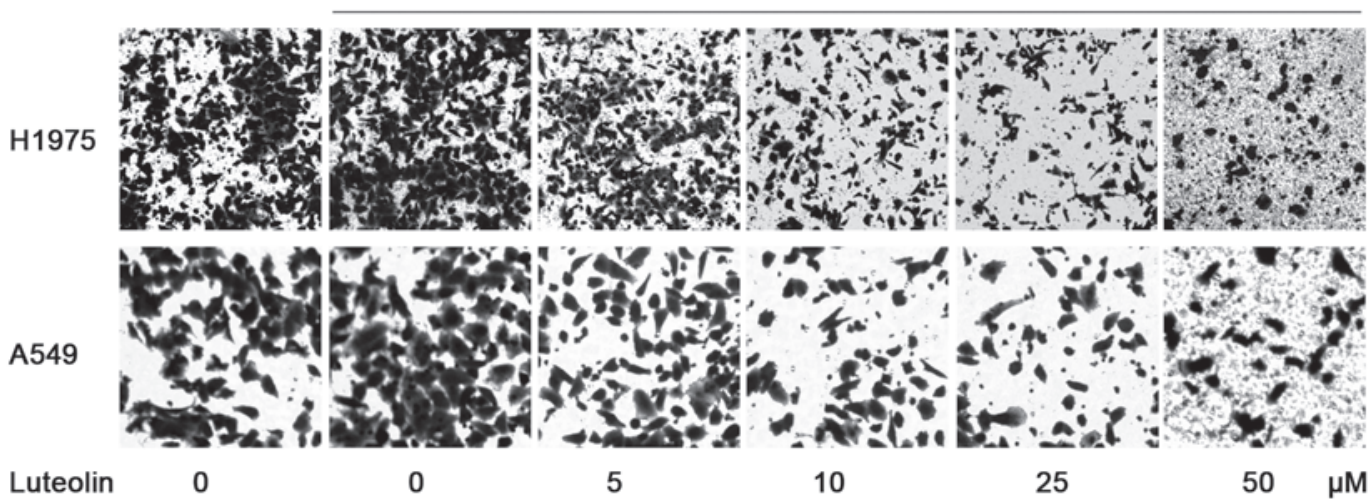

Figure 2. Inhibition of hypoxia-induced adhesion, motility and proliferation by luteolin in A549 and NCI-H1975 cells. (A) Hypoxic A549/H1975 cells were treated with the vehicle $(0.1 \%$ DMSO) or luteolin $(10-100 \mu \mathrm{M})$ for $24 \mathrm{~h}$, followed by the MTS assay. Quantitative results are presented as the means \pm SD ( $=3)$. ${ }^{*} \mathrm{P}<0.05 ;{ }^{* *} \mathrm{P}<0.01 ;{ }^{* * *} \mathrm{P}<0.001$ vs. control. (B and C) Normal and hypoxic A549/H1975 cells were treated with the vehicle (0.1\% DMSO) or luteolin (5-50 $\left.\mu \mathrm{M}\right)$ for $24 \mathrm{~h}$. The cells were trypsinized and seeded in HUVEC-coated and gelatin-coated 96-well plates in normal growth medium. After incubation for $1 \mathrm{~h}$, the cells were subjected to a cell adhesion assay. Quantitative results are presented as the means $\pm \mathrm{SD}(\mathrm{n}=3)$. "P<0.01 vs. normal group, ${ }^{*} \mathrm{P}<0.01 \mathrm{vs}$. control group. ( $\mathrm{D}$ and $\left.\mathrm{E}\right)$ Normal and hypoxic A549/H1975 cells were treated with the vehicle (0.1\% DMSO) or luteolin (5-50 $\mu \mathrm{M})$ for $24 \mathrm{~h}$ followed by Boyden chamber Transwell assays. The migrated cells were visualized and counted from 6 randomly selected fields (magnification, x200) using an inverted microscope. Representative images of migrated A549 and H1975 cells are shown in (E). Quantitative results are presented as the means \pm SD ( $=3$ ). ${ }^{*} \mathrm{P}<0.01$ vs.normal group, ${ }^{\#} \mathrm{P}<0.01$ vs.control group. DMSO, dimethylsulfoxide; MTS, 3-(4,5-dimethylthiazol-2-yl)-5-(3-carboxymethoxyphenyl)-2-(4-sulfophenyl)-2H-tetrazolium; SD, standard deviation.

regulatory circuits in the cells. Meanwhile, the morbidity and mortality in patients with cancer mainly originates from the formation of distant metastases. Recently, tremendous efforts have been taken to develop drugs that inhibit metastasis. 


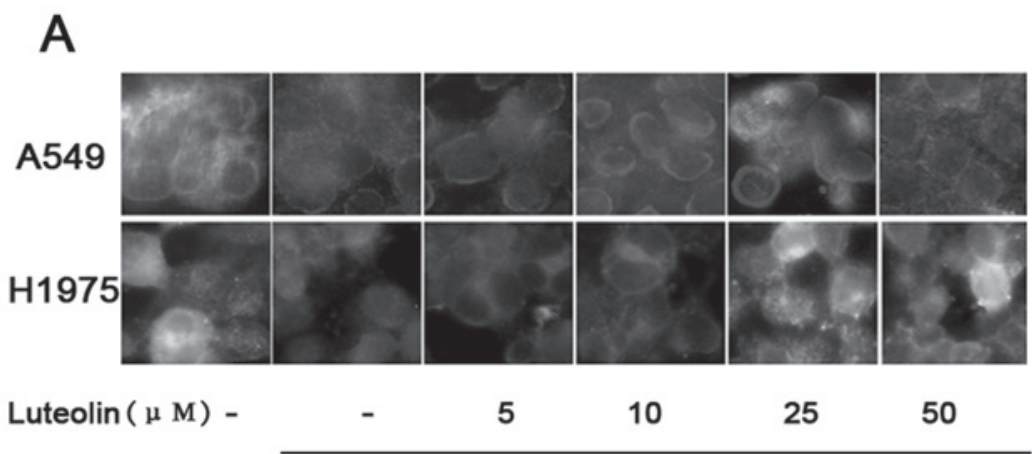

Hypoxia

B

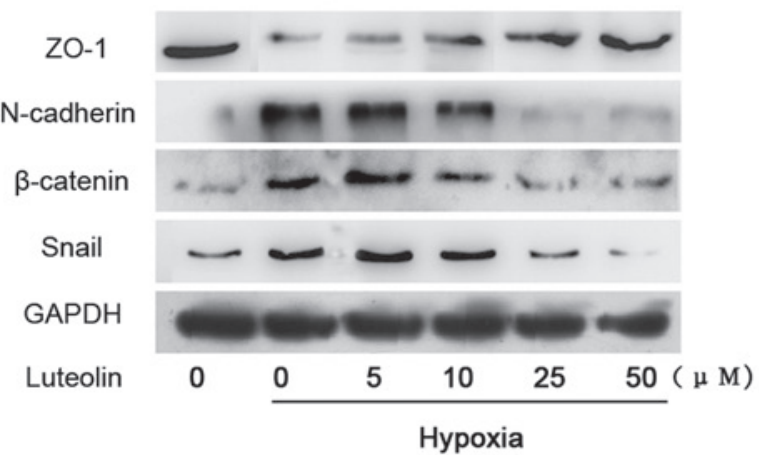

D

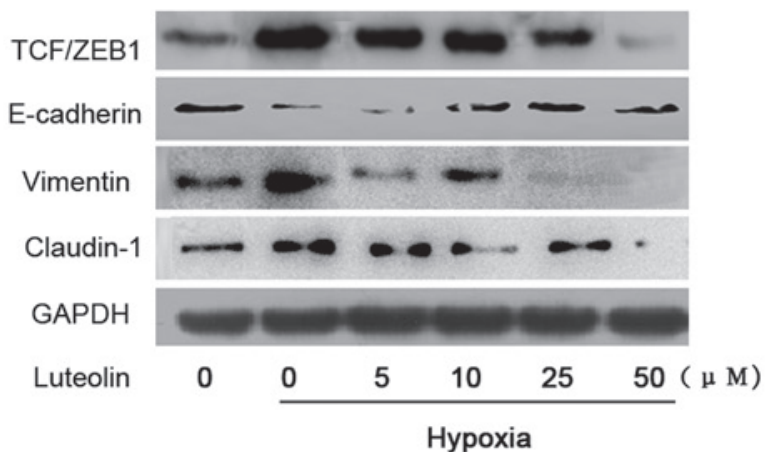

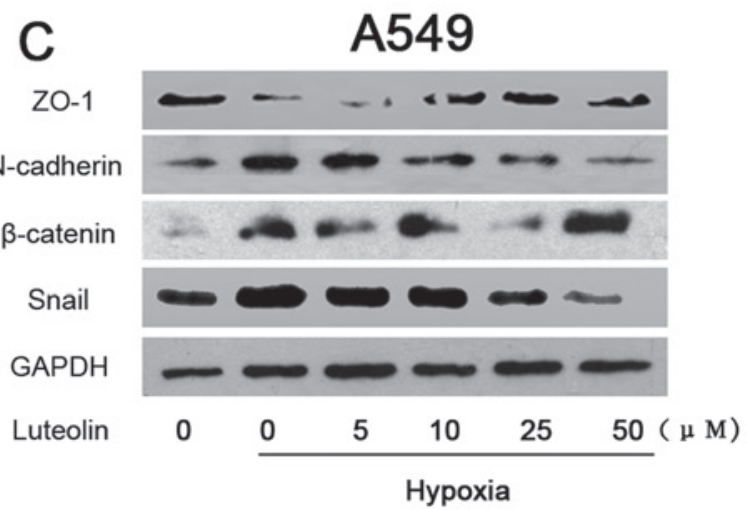

E

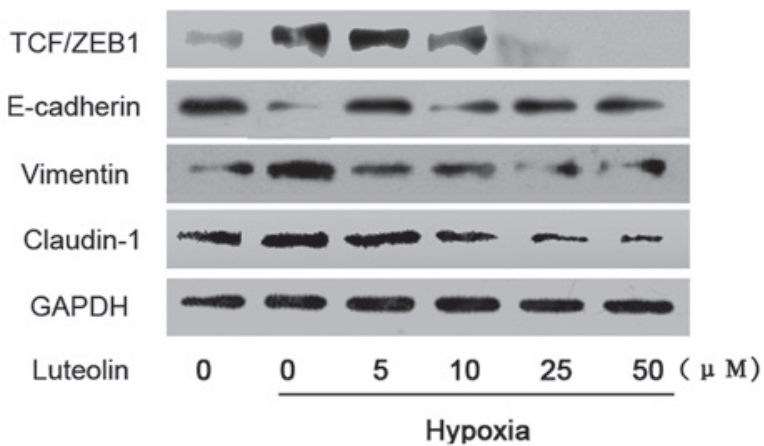

Figure 3. Inhibition of hypoxia-induced EMT by luteolin in A549 and NCI-H1975 cells. (A) Normal and hypoxic A549/H1975 cells were treated with the vehicle $(0.1 \% \mathrm{DMSO})$ or luteolin $(5-50 \mu \mathrm{M})$ for $24 \mathrm{~h}$, and E-cadherin protein level was then analyzed by an immunofluorescence assay. The other EMT-related proteins in the (B and D) NCI-H1975 cells and (C and E) A549 cells were then analyzed by western blot analysis with the indicated antibodies. GAPDH was used as the loading control. EMT, epithelial mesenchymal transition.

However, the identification of anti-metastatic agents remains unsuccessful at this point. It is widely recognized that tubular epithelial cells can differentiate into myofibroblasts via the EMT process, which plays an essential role in the development of NSCLC metastasis $(20,21)$. A phenotypic change causes the cells to become increasingly migratory and invasive (22). Moreover, EMT is the main cause of drug resistance $(23,24)$. Seeking effective drugs that suppress EMT has been a topic of intense research, and identifying herbal compounds or drug candidates that could effectively prevent or inhibit the EMT process is among the most attempted strategies in combating cancer. Traditional Chinese medicine has been widely utilized in the treatment of NSCLC. Therefore, identifying active ingredients in Chinese medicines that inhibit EMT could be instructive for the design and development of new anticancer agents.

Luteolin, a common active flavonoid compound, is found at high concentrations in Lonicera japonica, Hedyotis diffusa, and a number of other plants that have been widely exploited to treat NSCLC (25-27). The antitumor effect of luteolin has been documented in a number of types of human cancer. However, the precise effects of luteolin on invasion, migration and EMT, as well as the associated signaling pathways, have not been reported, particularly in NSCLC cells. In this study, for the first time, we demonstrate that luteolin inhibits hypoxiainduced EMT in NSCLC cells, supporting the hypothesis that 


\section{A}

NCl-H1975

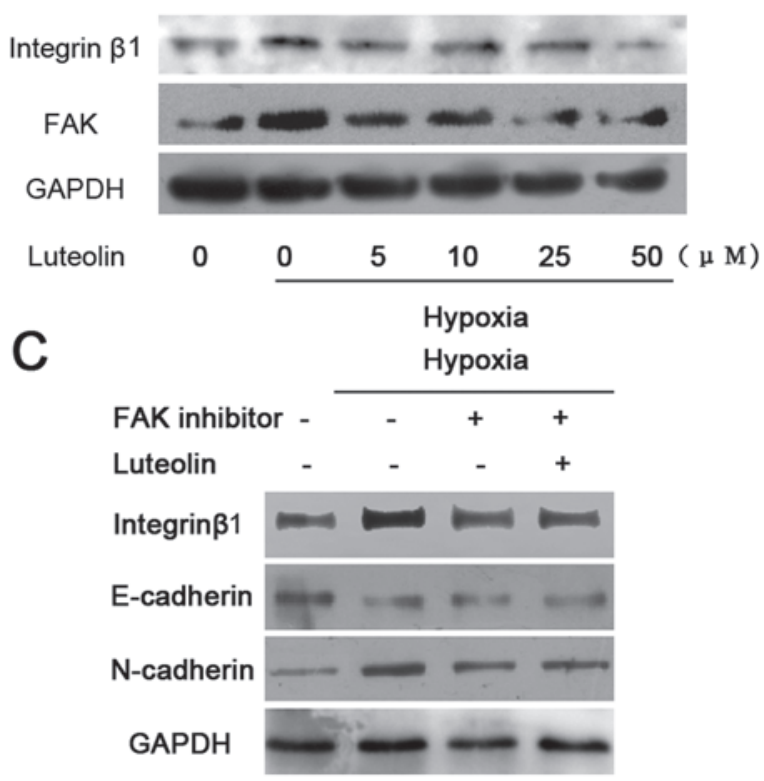

$\mathrm{NCl}-\mathrm{H} 1975$
B

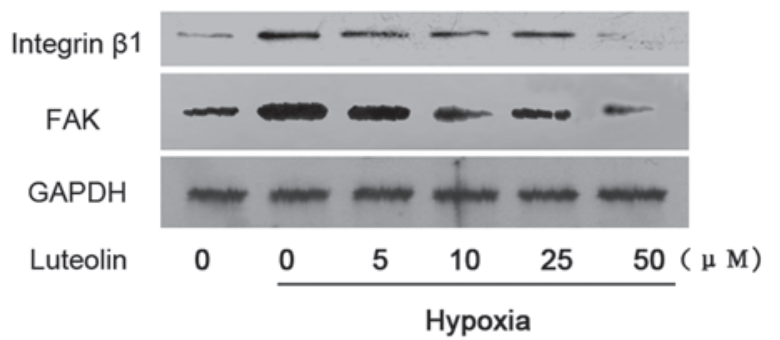

D

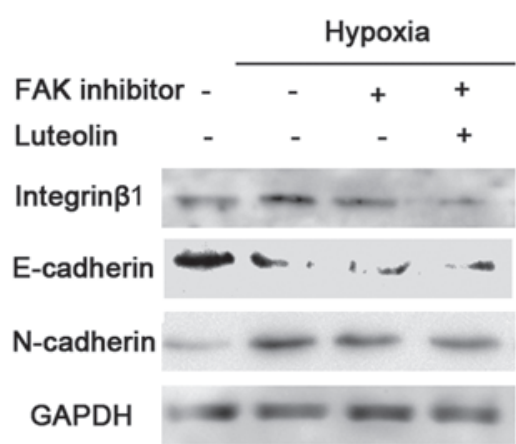

A549

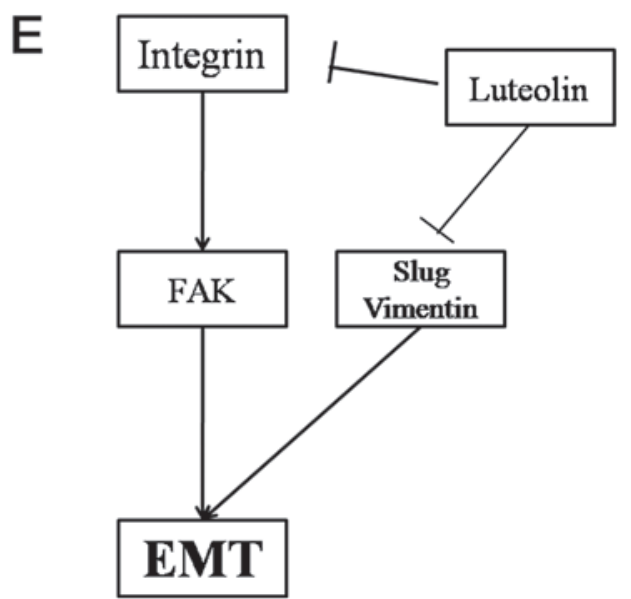

Figure 4. Inhibition of integrin $\beta 1 / F A K$ signaling pathways by luteolin in A549/NCI-H1975 cells. (A and B) Normal and hypoxic A549/H1975 cells were treated with the vehicle $(0.1 \%$ DMSO) or luteolin $(5-50 \mu \mathrm{M})$ for $24 \mathrm{~h}$ and the proteins were analyzed by western blot analysis with the indicated antibodies. GAPDH was used as the loading control. (C and D) Normal and hypoxic A549/H1975 cells were treated with the vehicle (0.1\% DMSO) or 50 $\mu \mathrm{M}$ luteolin plus $1 \mu \mathrm{M}$ FAK inhibitor for $24 \mathrm{~h}$. The cellular proteins were then analyzed by western blot analysis with the indicated antibodies. GAPDH was used as the loading control. (E) Possible molecular mechanism by which luteolin regulates EMT. FAK, focal adhesion kinase; EMT, epithelial mesenchymal transition; DMSO, dimethylsulfoxide.

luteolin is a potential anticancer agent, and that the use of this compound could be explored for the prevention and treatment of cancer metastasis.

In this study, we demonstrate that hypoxia suppresses the expression of E-cadherin and elevates that of $\mathrm{N}$-cadherin and vimentin, which was then attenuated by luteolin (Fig. 4), indicating the inhibition of hypoxia-induced EMT. Furthermore, our results showed that luteolin inhibited the hypoxia-induced cell morphological changes (EMT), cell proliferation, adhesion and motility associated with the EMT phenotype in hypoxic A549/H1975 cells. Mechanistically, the expression of integrin $\beta 1$ and FAK, which are critical proteins for EMT and cell motility, has been confirmed to be inhibited by luteolin (Fig. 4E). These results indicate that the activity of luteolin may account for its inhibitory effect on cancer metastasis, and its multiple anti-metastatic activities are potentially applicable to the design of anti-metastatic agents.

\section{Acknowledgements}

We thank Dr Shile Huang for critical reading and helpful discussions in preparing the manuscript. This study was supported in part by the National Natural Science Foundation of China (No. 81173174, 30772766), the Natural Science Foundation of Jiangsu Province (No. BK2010085, 2010562), the Educational Commission of Jiangsu Province (No. 09KJA360002), the Jiangsu College Graduate Research and Innovation Projects (2010-469 and 2010-471) and was a project of the Priority 
Academic Program Development of Jiangsu Higher Education Institutions (PAPD).

\section{References}

1. Jemal A, Bray F, Center MM, Ferlay J, Ward E and Forman D: Global cancer statistics. CA Cancer J Clin 61: 69-90, 2011.

2. Cataldo VD, Gibbons DL, Perez-Soler R and Quintas-Cardama A Treatment of non-small-cell lung cancer with erlotinib or gefitinib. N Engl J Med 364: 947-955, 2011.

3. Grimminger F, Schermuly RT and Ghofrani HA: Targeting non-malignant disorders with tyrosine kinase inhibitors. Nat Rev Drug Discov 9: 956-970, 2010

4. Kuner R, Muley T, Meister M, et al: Global gene expression analysis reveals specific patterns of cell junctions in non-small cell lung cancer subtypes. Lung Cancer 63: 32-38, 2009.

5. Le QT and Courter D: Clinical biomarkers for hypoxia targeting. Cancer Metastasis Rev 27: 351-362, 2008.

6. Hung JJ, Yang MH, Hsu HS, Hsu WH, Liu JS and Wu KJ: Prognostic significance of hypoxia-inducible factor-1alpha, TWIST1 and Snail expression in resectable non-small cell lung cancer. Thorax 64: 1082-1089, 2009.

7. Pine SR, Ryan BM, Varticovski L, Robles AI and Harris CC: Microenvironmental modulation of asymmetric cell division in human lung cancer cells. Proc Natl Acad Sci USA 107: 2195-2200, 2010.

8. Kim WY, Perera S, Zhou B, et al: HIF2alpha cooperates with RAS to promote lung tumorigenesis in mice. J Clin Invest 119: 2160-2170, 2009

9. Chowdhury AR, Sharma S, Mandal S, Goswami A, Mukhopadhyay S and Majumder HK: Luteolin, an emerging anti-cancer flavonoid, poisons eukaryotic DNA topoisomerase I. Biochem J 366: 653-661, 2002.

10. Hirano T, Higa S, Arimitsu J, et al: Luteolin, a flavonoid, inhibits AP-1 activation by basophils. Biochem Biophys Res Commun 340: 1-7, 2006.

11. Ueda H, Yamazaki $\mathrm{C}$ and Yamazaki M: Luteolin as an anti-inflammatory and anti-allergic constituent of Perilla frutescens. Biol Pharm Bull 25: 1197-1202, 2002.

12. Attoub S, Hassan AH, Vanhoecke B, et al: Inhibition of cell survival, invasion, tumor growth and histone deacetylase activity by the dietary flavonoid luteolin in human epithelioid cancer cells. Eur J Pharmacol 651: 18-25, 2011.

13. Amin AR, Wang D, Zhang H, et al: Enhanced anti-tumor activity by the combination of the natural compounds (-)-epigallocatechin-3-gallate and luteolin: potential role of p53. J Biol Chem 285: 34557-34565, 2010.

14. Lin YS, Tsai PH, Kandaswami CC, et al: Effects of dietary flavonoids, luteolin, and quercetin on the reversal of epithelialmesenchymal transition in A431 epidermal cancer cells. Cancer Sci 102: 1829-1839, 2011
15. Bagli E, Stefaniotou M, Morbidelli L, et al: Luteolin inhibits vascular endothelial growth factor-induced angiogenesis; inhibition of endothelial cell survival and proliferation by targeting phosphatidylinositol 3'-kinase activity. Cancer Res 64: 7936-7946, 2004.

16. Lin Y, Shi R, Wang X and Shen HM: Luteolin, a flavonoid with potential for cancer prevention and therapy. Curr Cancer Drug Targets 8: 634-646, 2008

17. Gupta R, Chetty C, Bhoopathi P, et al: Downregulation of uPA/uPAR inhibits intermittent hypoxia-induced epithelial-mesenchymal transition (EMT) in DAOY and D283 medulloblastoma cells. Int J Oncol 38: 733-744, 2011.

18. Johnson FM, Saigal B, Talpaz M and Donato NJ: Dasatinib (BMS-354825) tyrosine kinase inhibitor suppresses invasion and induces cell cycle arrest and apoptosis of head and neck squamous cell carcinoma and non-small cell lung cancer cells. Clin Cancer Res 11: 6924-6932, 2005.

19. Shah PP, Fong MY and Kakar SS: PTTG induces EMT through integrin alpha(V)beta(3)-focal adhesion kinase signaling in lung cancer cells. Oncogene: Nov 14, 2011 (Epub ahead of print).

20. Blanco E, Bey EA, Khemtong C, et al: Beta-lapachone micellar nanotherapeutics for non-small cell lung cancer therapy. Cancer Res 70: 3896-3904, 2010.

21. Tauler J, Zudaire E, Liu H, Shih J and Mulshine JL: hnRNP A2/B1 modulates epithelial-mesenchymal transition in lung cancer cell lines. Cancer Res 70: 7137-7147, 2010.

22. Yilmaz M and Christofori G: EMT, the cytoskeleton, and cancer cell invasion. Cancer Metastasis Rev 28: 15-33, 2009

23. Thomson S, Buck E, Petti F, et al: Epithelial to mesenchymal transition is a determinant of sensitivity of non-small-cell lung carcinoma cell lines and xenografts to epidermal growth factor receptor inhibition. Cancer Res 65: 9455-9462, 2005.

24. Yauch RL, Januario T, Eberhard DA, et al: Epithelial versus mesenchymal phenotype determines in vitro sensitivity and predicts clinical activity of erlotinib in lung cancer patients. Clin Cancer Res 11: 8686-8698, 2005.

25. Leung HW, Hour MJ, Chang WT, et al: P38-associated pathway involvement in apoptosis induced by photodynamic therapy with Lonicera japonica in human lung squamous carcinoma $\mathrm{CH} 27$ cells. Food Chem Toxicol 46: 3389-3400, 2008.

26. You BJ, Wu YC, Bao BY, et al: Rottlerin inhibits Lonicera Japonica-induced photokilling in human lung cancer cells through cytoskeleton-related signaling cascade. Evid Based Complement Alternat Med 2011: 193842, 2011.

27. Lee HZ, Bau DT, Kuo CL, Tsai RY, Chen YC and Chang YH: Clarification of the phenotypic characteristics and anti-tumor activity of Hedyotis diffusa. Am J Chin Med 39: 201-213, 2011. 